\title{
Sequential Amine-Functionalization Inducing Structural Transition in Aldehyde-Containing Zeolitic Imidazolate Framework: Application to Gas Separation Membranes
}

Fernando Cacho-Bailo, ${ }^{a}$ Miren Etxeberría-Benavides, ${ }^{\mathrm{b}}$ Oğuz Karvan, ${ }^{\mathrm{b}}$ Carlos Téllez, ${ }^{\mathrm{a}}$ Joaquín Coronas ${ }^{\mathrm{a},{ }^{*}}$

${ }^{a}$ Chemical and Environmental Engineering Department and Instituto de Nanociencia de Aragón (INA), Universidad de Zaragoza, 50018 Zaragoza, Spain

${ }^{b}$ Tecnalia Research and Innovation, Energy and Environmental Division, 20009 Donostia-San Sebastián, Spain

Corresponding Author: *coronas@unizar.es

Electronic supplementary information (ESI) available: TG analyses of the amine-functionalized SIM-1 powders and FTIR spectra of the $\mathrm{P} 84^{\circledR}$ reaction with amines.

\begin{abstract}
$\underline{\text { Abstract }}$
A modification in the gas separation performance of zeolitic imidazolate framework (ZIF)-supported hollow fiber (HF) membranes by means of an imine-condensation functionalization reaction carried out by microfluidics is reported. The accommodation of voluminous amine molecules in the SIM-1, Zn(4-methyl-5imidazolcarboxaldehyde) $)_{2}$, also known as ZIF-94, sod structure during the functionalization reaction caused the ZIF atoms to be rearranged in a less dense rho structure, with a wider pore diameter and a diminished $\mathrm{CO}_{2}$ affinity. These changes had effects on the membrane performance, resulting in an enhanced $\mathrm{CO}_{2}$ permeance while maintaining a good permeance-selectivity balance. ZIF aldehyde-containing SIM-1 membranes were early prepared on the inner side of polymeric P $84^{\circledR}$ HF using a microfluidic approach. The SIM-1 membranes displayed very interesting results in the separation of gas mixtures of great relevance in the natural gas field. High selectivities in the separation of $\mathrm{He} / \mathrm{CH}_{4}$ (160), $\mathrm{H}_{2} / \mathrm{CH}_{4}$ (136) and $\mathrm{CO}_{2} / \mathrm{CH}_{4}$ (38) mixtures were achieved, the first SIM-1 membranes with such a high separation performance to the best of our knowledge. These SIM-1 membranes were in situ stepwise functionalized with long-chain amine solutions, namely, hexyl- and nonylamine. Microfluidics allowed the easy sequential implementation of this post-reaction step in
\end{abstract}


the membrane fabrication procedure. An imine-condensation reaction took place between the aldehyde groups in the 4-methyl-5-imidazolcarboxaldehyde ligand forming the SIM-1 and the corresponding amines. The extent of the reaction was analyzed by FTIR, TGA and XRD, together with the changes in the textural properties and the adsorption capacities.

\section{$\underline{\text { Keywords }}$}

MOF, amine, functionalization, membrane, gas separation

\section{$\underline{\text { 1. Introduction }}$}

Metal-organic frameworks (MOFs) offer novel chemical versatility as a result of the extensive supply of organic moieties in their forming ligands. ${ }^{1,2}$ This versatility, which had not been previously observed in other inorganic microporous materials such as zeolites, ${ }^{3}$ is potentially useful in numerous applications. These include gas separation ${ }^{4}$ and adsorption, ${ }^{1,5,6}$ catalysis $^{7,8}$ and controlled drug delivery. ${ }^{9}$ A large number of studies have taken advantage of this MOF resourcefulness: among others, Thompson et al. ${ }^{10,11}$ and Eum et $a l^{12}$ described the preparation of hybrid mixed-ligand MOFs and the resulting tune in their porosities, whereas Marti et al. ${ }^{13}$ and Fei et al. ${ }^{14}$ reported post-synthetic organic ligand and metal cluster exchanges from solution, respectively. Moreover, the covalent functionalization reaction is perhaps the most widespread strategy for MOF post-modification. ${ }^{15,16}$ Most of these studies report gas adsorption equilibria, but nevertheless lack diffusion data through the MOF structures, which are needed for prediction of post-modified separation selectivities. ${ }^{17}$

An interesting combination of narrow porosity and chemical versatility can be found in aldehyde groupcontaining ZIFs (zeolitic imidazolate frameworks, a subclass of MOFs), such as ZIF-90 (Zn-imidazole-2carboxaldehyde), ${ }^{1}$ SIM-1 (from Substituted-Imidazolate-Material-1, ${ }^{18,19}$ also known as ZIF-94 ${ }^{20}$ ) and ZIF-93 (both SIM-1 and ZIF-93 have the formula Zn-4-methyl-5-imidazolecarboxaldehyde), ${ }^{21}$ among others. These ZIFs crystallize in the zeolite-like structures sod (ZIF-90 and SIM-1) and rho (ZIF-93). Because of their narrow and highly-constricted limiting pore diameters, the properties of these and other ZIFs in their assynthesized form have been widely studied in separation processes. ${ }^{18,22-24}$ Furthermore, their aldehyde groups can be functionalized to modify the pore sizes and textural and adsorptive properties of the material. ${ }^{25}$ Morris et al. reacted ZIF-90 powder with ethanolamine, ${ }^{26}$ demonstrating the potential use of MOF crystals as discrete reactive molecules. ${ }^{27}$ Huang applied this concept to the membrane field, post-modifying a flat inorganicsupported ZIF-90 membrane. ${ }^{28}$ This MOF was first reduced (aldehyde to alcohol) with $\mathrm{NaBH}_{4}$ to give rise to ZIF-91. Meanwhile, an imine-condensation reaction with ethanolamine led to the so-called ZIF-92, which provided a significant increase in the $\mathrm{H}_{2} / \mathrm{CO}_{2}$ separation selectivity. $\mathrm{Yu}$ et al. coated a chromatographic capillary column in a similar manner with APTES-functionalized ZIF-90. ${ }^{29}$ The same idea was applied by Aguado et al. in the post-synthetic functionalization of SIM-1 with dodecylamine that increased the hydrophobicity of SIM-1-coated alumina spheres. ${ }^{30}$ 
The as-synthesized MOF ZIF-90 was afterwards used by Brown et al. ${ }^{31}$ in the fabrication of outer-supported MOF hollow fiber (HF) membranes, prior to another work on the fabrication of inner-supported ZIF-8 TORLON $^{\circledR}$ HF membranes by microfluidics. ${ }^{32}$ To make the synthesis procedure compatible with the polymeric supports, light alcohols and mild conditions were used as substitutes for DMF or other harassing solvents. ${ }^{10,29,33,34}$ While only sod-ZIF-90 has been crystallized using imidazole-2-carboxaldehyde ligand, both SIM-1 (sod) and ZIF-93 (rho) can be formed with 4-methyl-5-imidazolecarboxaldehyde depending on the synthesis procedure. ${ }^{19-21}$ Although some authors have claimed that imidazole with substituted positions 4 and 5 preferentially forms a ZIF material having the rho structure, ${ }^{21,35-37}$ crystallization can be positively directed into the SIM-1 form, ${ }^{18-20,34,38}$ whose crystalline structure was elucidated in $2015 .{ }^{39}$ However, although a superior $\mathrm{CO}_{2}$ adsorption affinity has been reported, ${ }^{18,20}$ its use in membranes as a molecular sieve for gas separation remains uncommon and provided only a modest performance. ${ }^{18}$

The covalent functionalization of MOF structures can be easily implemented in the fabrication of inner-MOFsupported HF membranes by microfluidics, as has recently been achieved in other approaches as the fabrication of a bimetallic two-layered ZIF membrane. ${ }^{40}$ MOF crystallization, post-functionalization and washing steps can be sequenced ${ }^{41}$ in the desired conditions to tune the membrane to achieve, for instance, a specific MOF pore size or particular surface textural properties. ${ }^{25}$ In this manner, MOF versatility under mild conditions can be exploited, together with the inherent advantages of the microfluidic fabrication of HF membranes. These include a continuous ecofriendly synthesis procedure with significant solvent and reagent savings and the protection of the MOF layer. ${ }^{17,42} \mathrm{HF}$ polymeric supports, easy to extrude and shape, are an efficient and compact conformation for membranes. ${ }^{43,44}$ They also provide high contact surface to volume ratios and an enhanced compatibility with MOF materials because of their shared organic character. ${ }^{45}$

In this work, SIM-1 supported HF membranes exhibiting a high performance in the separation of gas mixtures with relevance in the natural gas field were prepared by microfluidics. The imine-condensation aldehydefunctionalization reaction within these SIM-1@P84 ${ }^{\circledR} \mathrm{HF}$ membranes was then applied as a flexible, easilycontrollable additional step in the microfluidic synthesis procedure. The derived effects of the functionalization with two different amines (hexyl- and nonylamine) were first monitored on the bulk SIM-1 powder and then on the gas separation performance of the membranes by different techniques. An alteration of the MOF crystalline structure and a parallel reaction with the co-polyimide P84 ${ }^{\circledR}$ support were also observed.

\section{$\underline{\text { 2. Experimental }}$}

\section{$\underline{\text { 2.1 Membrane fabrication }}$}

The SIM-1@P84 ${ }^{\circledR}$ HF membranes were fabricated through the microfluidic setup used in our previous works. $^{22,42} \quad{\mathrm{P} 84^{\circledR}}^{\circledR} \quad$ (BTDA-TDI/MDI, 3,3'4,4'-benzophenone tetracarboxylic dianhydride, 80\% methylphenylene-diamine $+20 \%$ methylene diamine) co-polyimide hollow fiber supports (202 $\mu \mathrm{m} \mathrm{ID,} 356$ 
$\mu \mathrm{m}$ OD) were fabricated by a procedure described elsewhere. ${ }^{45}$ These P8 $4{ }^{\circledR}$ HF supports were intentionally fabricated highly permeable to be used as supports for membrane fabrication.

First, a $40 \mathrm{~cm}$-long HF support was joined to the PTFE tubing (0.04 in ID, 1/16 in OD) of the microfluidic plant by means of a PTFE-intended glue. Three microfluidic solutions were prepared and loaded into $20 \mathrm{~mL}$ PP syringes, namely, pure deionized water, a $0.10 \mathrm{~mol} \cdot \mathrm{L}^{-1}$ solution of $\mathrm{ZnNO}_{3} \cdot 6 \mathrm{H}_{2} \mathrm{O}$ (zinc nitrate hexahydrate, Sigma-Aldrich, $98 \%$ ) in deionized water (metal solution) and a $0.25 \mathrm{~mol} \cdot \mathrm{L}^{-1} 4 \mathrm{~m} 5 \mathrm{ImCA}$ (4-methyl-5imidazolcarboxaldehyde, $\mathrm{C}_{5} \mathrm{H}_{6} \mathrm{~N}_{2} \mathrm{O}$, Sigma-Aldrich, $99 \%$ ) and $0.13 \mathrm{~mol} \cdot \mathrm{L}^{-1} \mathrm{NaCOOH}$ (sodium formate, Sigma-Aldrich, $>99 \%$ ) solution in a 50/50 v/v deionized water/absolute ethanol mixture (ligand solution).

The HF support was inner soaked and washed with $2 \mathrm{~mL}$ of deionized water at an $80 \mu \mathrm{L} \cdot \mathrm{min}^{-1}$ flow rate, prior to the start of the SIM-1 growth. The metal and ligand solutions were then injected together at room temperature at an $80 \mu \mathrm{L} \cdot \mathrm{min}^{-1}$ flow rate $\left(40 \mu \mathrm{L} \cdot \mathrm{min}^{-1}\right.$ each) from the syringes using microfluidic pumps (NE300, New Era Pump Systems) into the HF lumen. The mixture of the metal and ligand solutions took place in a T-junction at the entrance of the HF support. The SIM-1 synthesis lasted $2.5 \mathrm{~h}$ and a total volume of $12 \mathrm{~mL}$ (6 $\mathrm{mL}$ each) was pumped. Finally, $5 \mathrm{~mL}$ of deionized water at an $80 \mu \mathrm{L} \cdot \mathrm{min}^{-1}$ flow rate was pumped inside the HF membrane lumen to wash and drag unattached MOF particles. A $20 \mathrm{~cm}$-long piece was cut from the entire membrane (SIM-1@P84 $4^{\circledR}$ ) and dried at room temperature.

\section{$\underline{\text { 2.2 Membrane post-functionalization with amines }}$}

The $20 \mathrm{~cm}$-long SIM-1@P84 ${ }^{\circledR}$ piece of membrane remaining was then immediately post-functionalized at room temperature. A $0.2 \mathrm{~mol} \cdot \mathrm{L}^{-1}$ solution of nonylamine $\left(\mathrm{C}_{9} \mathrm{H}_{21} \mathrm{~N}\right.$, Sigma-Aldrich, $\left.98 \%\right)$ in absolute ethanol was injected into the membrane lumen for $2 \mathrm{~h}$, totalizing a volume of $10 \mathrm{~mL}$. Finally, $5 \mathrm{~mL}$ of absolute ethanol at an $80 \mu \mathrm{L} \cdot \mathrm{min}^{-1}$ flow rate was pumped inside the HF membrane lumen to wash the unreacted nonylamine, and the resulting C9@SIM-1@P84 ${ }^{\circledR}$ membrane was dried at room temperature. The same procedure was followed with a hexylamine $\left(\mathrm{C}_{6} \mathrm{H}_{15} \mathrm{~N}\right.$, Sigma-Aldrich, $\left.99 \%\right)$ solution in deionized water to obtain a C6@SIM-1@P84 ${ }^{\circledR}$ membrane.

The solution stream was continuously collected at the end of the HF. The SIM-1 powder was centrifuged at $10,000 \mathrm{rpm}$ for $15 \mathrm{~min}$ and thoroughly washed with deionized water. A portion was then dispersed in the corresponding $0.2 \mathrm{~mol} \cdot \mathrm{L}^{-1}$ amine solution for functionalization for $2 \mathrm{~h}$. The functionalized SIM-1 powders were finally centrifuged under the same conditions, washed and dried overnight at $100^{\circ} \mathrm{C}$. Pure SIM-1, C9@SIM-1 and C6@SIM-1 powders were used for characterization, on the assumption that the MOF forming part of the membranes had been synthesized in an identical manner. A portion of the SIM-1 dispersion was allowed to react for $24 \mathrm{~h}$ under the same conditions, giving rise to the C9_24h@SIM-1 sample. Figure 1 shows a scheme of the imine-condensation reaction taking place in the aldehyde pendant groups in the SIM-1 during its functionalization, on this occasion with nonylamine. 


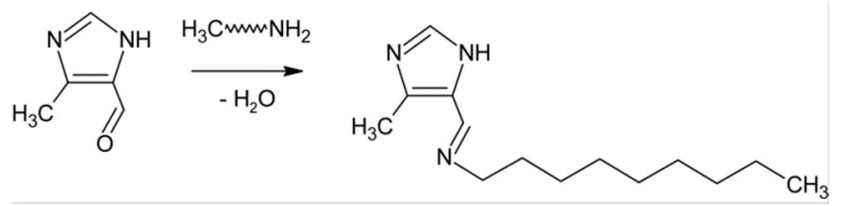

Figure 1. Imine-condensation SIM-1 functionalization scheme. Aldehyde groups in the 4m5ImCA ligands in the SIM-1 structure react with the amine (in this case nonylamine) to form an imine with a pendant hydrocarbon chain.

\section{$\underline{2.3 \text { Characterization \& permeation testing }}$}

XRD (X-ray diffraction) patterns were obtained using a D-Max Rigaku X-ray diffractometer (40 kV, $80 \mathrm{~mA})$ with a $\mathrm{Cu} K \alpha(\lambda=0.1542 \mathrm{~nm})$ rotating anode from 3 to $36^{\circ}(2 \theta)$ and a $0.025^{\circ} \cdot \mathrm{s}^{-1}$ step. TGA (thermogravimetric analyses) were carried out with a Mettler Toledo TGA/DSC SF/755, oxidizing the samples in an air atmosphere from 25 to $850{ }^{\circ} \mathrm{C}$ at a rate of $10^{\circ} \mathrm{C} \cdot \mathrm{min}^{-1}$. ATR-FTIR (attenuated total reflection-Fourier transform infrared spectroscopy) spectra were obtained using a Bruker Vertex 70, accumulating 40 scans from 4000 to $600 \mathrm{~cm}^{-1}$ with $4 \mathrm{~cm}^{-1}$ resolution. $\mathrm{N}_{2}$ adsorption isotherms at $77 \mathrm{~K}$ were obtained using a Micromeritics Tristar, and $\mathrm{CO}_{2}$ adsorption isotherms at $298 \mathrm{~K}$ with a Micromeritics ASAP 2020. Samples were previously degassed at $175^{\circ} \mathrm{C}$ for $8 \mathrm{~h}$. SEM (scanning electron microscopy) images were obtained with a FEI INSPECTF operated at $15 \mathrm{kV}$.

Permeation testing of the membranes was carried out using the Wicke-Kallenbach technique, the MOF@HF membranes being sealed with an epoxy resin in a 1/8-inch stainless steel-tubing module, as described elsewhere. ${ }^{40,42}$ All permeation tests in this work were carried out at $35^{\circ} \mathrm{C}$, the temperature being controlled with a convective oven. Gas mixtures were fed inside the HF lumen, in contact with the MOF layer, whereas the permeate stream was swept with Ar and analyzed with a MicroGC Agilent 3000A.

\section{Results and Discussion}

\subsection{SIM-1@P84 ${ }^{\circledR}$ membranes by microfluidics}

High-quality SIM-1@P84 ${ }^{\circledR}$ HF membranes were prepared with our microfluidic setup. This demonstrates the generalization of the method, as in our recent works concerning ZIF-7, ZIF-8, ZIF-93 and ZIF-9. ${ }^{22,40,42}$ Minor changes in the versatile microfluidic synthesis can give rise to different MOF inner-supported HF membranes. In this case, the water/ethanol solvent combination drove the crystallization towards the sod-structure of the SIM-1, denser than the rho of the ZIF-93. ${ }^{20}$ These are also the first membranes with the ZIF SIM-1 showing such a high gas separation performance to the best of our knowledge.

Figure 2 shows the SEM images of the bulk (a) and microfluidic (b) synthesized SIM-1 powdered-samples. A very wide particle size distribution was observed in the MOF collected during the fabrication of the SIM-1 membranes: each portion of the SIM-1 dispersion volume had a different crystallization-reaction time from 0 to $2.5 \mathrm{~h}$, leading to heterogeneous crystal sizes of SIM-1. A compact and continuous $7.1 \pm 0.4 \mu \mathrm{m}$ SIM-1 
layer was observed on the inner surface of the resulting microfluidic-fabricated P8 $44^{\circledR} \mathrm{HF}$ membranes (Figure 2c-d). FTIR, XRD and TGA results together with the gas adsorption capacity of the SIM-1 powder are shown in Figures 3 to 5 and S1, respectively. A microporous structure in the SIM-1 powder can be deduced from its IUPAC type I isotherm, with a specific surface area (SSA) of $591 \mathrm{~m}^{2} \cdot \mathrm{g}^{-1}$ and a pore volume of $0.31 \mathrm{~cm}^{3} \cdot \mathrm{g}^{-1}$, in agreement with those previously reported for this material. ${ }^{18,20}$

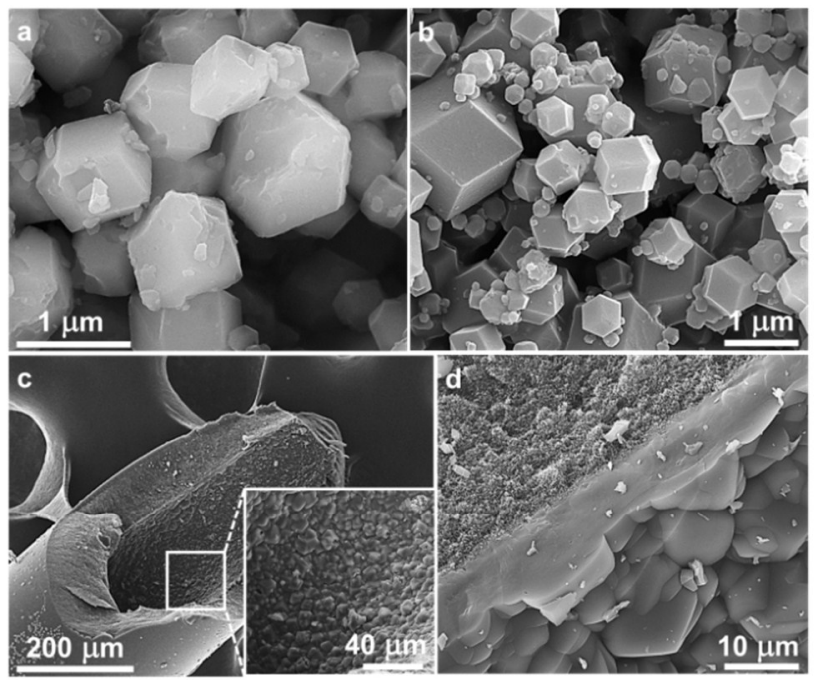

Figure 2. SEM images of as-synthesized SIM-1 powder fabricated in batch (a) and that collected during the microfluidic HF membrane fabrication (b). Images (c) and (d) show the fabricated SIM-1@P84 ${ }^{\circledR}$ HF membranes at different magnifications. A continuous inner-supported SIM-1 layer can be observed.

The constricted pores of SIM-1, with voluminous methyl- and aldehyde pendant groups in positions 4 and 5 in the imidazolate ring, led to good results in the gas separation tests. Averaged gas permeances and separation selectivities are shown in Table 1. The mixtures used during the tests to be separated in the membrane are of great interest in the natural gas field, i.e. $\mathrm{H}_{2} / \mathrm{CH}_{4}$ and $\mathrm{He} / \mathrm{CH}_{4}$ for hydrogen and helium recovery and $\mathrm{CO}_{2} / \mathrm{CH}_{4}$ for natural gas purification. ${ }^{46}$ Attractive high selectivities were obtained. An effective molecular sieving mechanism through the SIM-1 porous structure was deduced. The relatively low gas permeances provided were related to the continuous MOF layers with an absence of defects or pinholes (Figure 2d) but also with constricted gas diffusion through the MOF material. Moreover, the good adherence shown in the SEM images suggests a high SIM-1 - P84 $4^{\circledR}$ polymer compatibility.

Table 1. Averaged permeance and selectivity results obtained in the gas mixture permeation tests at $35{ }^{\circ} \mathrm{C}$ using pure SIM-1@P84 ${ }^{\circledR}$ fabricated membranes.

\begin{tabular}{|c|c|c|c|c|c|c|}
\hline \multirow{2}{*}{ Gas } & \multirow{2}{*}{$\begin{array}{c}\text { Kinetic } \\
\text { diameter }[\AA ̊]\end{array}$} & \multicolumn{2}{|c|}{ Permeance } & \multicolumn{3}{|c|}{ Selectivity } \\
\hline & & {$\left[\mathrm{mol} \cdot \mathrm{m}^{-2} \cdot \mathrm{s}^{-1} \cdot \mathrm{Pa}^{-1}\right]$} & [GPU] & $\mathrm{H}_{2}$ & $\mathrm{CO}_{2}$ & $\mathrm{CH}_{4}$ \\
\hline $\mathrm{He}$ & 2.6 & $5.0 \pm 2.5 \cdot 10^{-9}$ & $15.0 \pm 7.5$ & $1.2^{a}$ & $4.3^{a}$ & $160 \pm 14$ \\
\hline $\mathrm{H}_{2}$ & 2.9 & $4.2 \pm 2.2 \cdot 10^{-9}$ & $12.7 \pm 6.5$ & & $3.6 \pm 0.0$ & $136 \pm 1.0$ \\
\hline
\end{tabular}




\begin{tabular}{l|l|c|c|l|l|l|}
\hline $\mathrm{CO}_{2}$ & 3.3 & $1.2 \pm 0.6 \cdot 10^{-9}$ & $3.5 \pm 1.8$ & & & $37.7 \pm 0.8$ \\
\hline $\mathrm{CH}_{4}$ & 3.8 & $3.1 \pm 1.5 \cdot 10^{-11}$ & $0.1 \pm 0.0$ & & & \\
\hline
\end{tabular}

${ }^{a}$ Ideal selectivities calculated as the ratio of gas permeance data in the studied mixtures.

Therefore, a functionalization reaction involving the pendant aldehyde group in SIM-1 was sequentially implemented in the microfluidic setup to modify the gas permeation performance of the fabricated membranes.

\subsection{Powdered SIM-1 imine-condensation functionalization}

The inner-supported MOF layers on the P84 ${ }^{\circledR}$ HF support to be functionalized were reacted with an amine solution immediately after their growth and washing, as indicated in the Experimental section. Identical procedures were applied to the powders collected during the corresponding syntheses in order to monitor the progress of the reaction.

Figures 3 and S1 show the FTIR spectra and TGA curves, respectively, of the functionalized SIM-1 powders, from which the extent of the reaction could be deduced. A progressive diminishing on the aldehyde band $\left(1660 \mathrm{~cm}^{-1}\right)$ with respect to that of the imine $\left(1622 \mathrm{~cm}^{-1}\right)$, together with the appearance of a band relating to the C-H bonding of the aliphatic chain (around $2900 \mathrm{~cm}^{-1}$ ) was observed as a function of the extent of the functionalization reaction. Qualitatively, it can be stated that only the SIM-1 treated with nonylamine for $24 \mathrm{~h}$ achieved a full conversion of its aldehyde groups (sample C9_24h@SIM-1), while powdered samples C6@ and C9@SIM-1 were only partially functionalized.

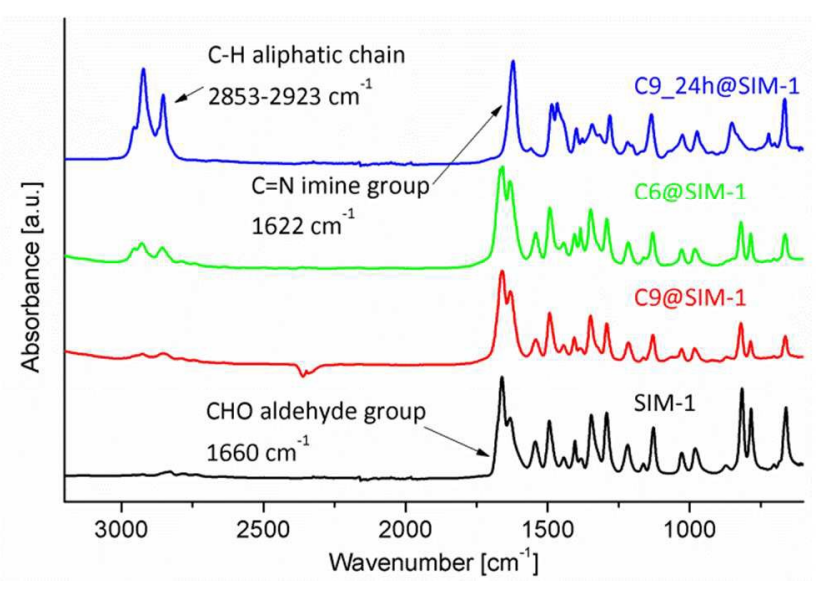

Figure 3. ATR-FTIR spectra of the powdered pure SIM-1 and amine-functionalized SIM-1 samples. A complete functionalization reaction could only be deduced in the C9_24h@SIM-1 sample, while 2 h-functionalization of the C6 and C9-amines led to partial conversions.

Besides, a quantitative reaction extent was estimated from TGA using the normalized experimental residues at $750{ }^{\circ} \mathrm{C}$. Table 2 shows a $43 \%$ functionalization extent in sample C6@SIM-1, while only a slight reactivity could be deduced from C9@SIM-1 (6.8 \%). Hexylamine, water soluble and with a shorter hydrocarbon chain 
than nonylamine, could penetrate deeper in the SIM-1 porous structure and react, giving rise to almost half of the aldehyde groups being converted into imine-bonded aliphatic pendant chains. In contrast, ethanoldissolved nonylamine, heavier and larger to penetrate in the MOF pores, led to a minor extent of the functionalization during the same time. These low reaction rates and partial conversions, assumed to have taken place from the surface to the bulk material, were positive because they hindered the amorphization of the material, as shown in Figure 4 for sample C9_24h@SIM-1. The residue of the pure SIM-1 powder at 750 ${ }^{\circ} \mathrm{C}$ fitted with that expected from its composition.

Table 2. Extent of the SIM-1 amine-functionalization reaction calculated from the TG analyses.

\begin{tabular}{c|c|c|c|} 
& \multicolumn{1}{c}{$\begin{array}{c}\text { TGA } \\
\text { residue }^{a}[\mathrm{wt} \%]\end{array}$} & $\begin{array}{c}\text { Expected } \\
\text { residue }^{b}[\mathrm{wt} \%]\end{array}$ & $\begin{array}{c}\text { Functionalization } \\
\text { Extent [\%] }\end{array}$ \\
\hline SIM-1 & 30.1 & 28.7 & 0 \\
\hline C9@SIM-1 & 29.4 & 19.9 & 6.8 \\
\hline C6@SIM-1 & 26.7 & 22.2 & 43 \\
\hline C9_24h@SIM-1 & 16.1 & 19.9 & $>100$ \\
\hline
\end{tabular}

${ }^{a}$ Normalized residue in weight of the samples after oxidation in air at $750{ }^{\circ} \mathrm{C}$.

${ }^{b}$ Expected residue for a $100 \%$ functionalization reaction extent of the corresponding amine with SIM-1 (i.e. every pending aldehyde group converted into imine).

At the same time, XRD spectra unexpectedly revealed a structural alteration deriving from the amine reaction with SIM-1. A sod transformation into the rho crystalline phase was clearly observed (Figure 4), proportional to the extent of the reaction and the amine weight gain monitored by TGA and concomitant with some loss of crystallinity in the case of the $24 \mathrm{~h}$ reaction with nonylamine. The accommodation of voluminous amine molecules in the SIM-1 structure during the functionalization reaction, playing perhaps a template role, drove the ZIF atoms to be rearranged in a less dense crystalline phase structure. The rho structure, formed by the combination of Ita and $\mathbf{d 8 r}$ composite building units, displays a higher cavity diameter $(17.9 \AA)^{20}$ with 8 member ring apertures. This crystalline conformation can more easily accommodate the voluminous amine molecules than sod, built from sod cages with 4- and 6-member ring access windows and displaying a cavity diameter of $7.5 \AA,{ }^{39}$ as observed in Figure 4. A similar solution-mediated phase transition was reported within a Co-NIm ZIF material by Biswal et al., where the size of the solvent used played an important role. ${ }^{37}$ Herein, a longer-extended hexylamine reaction led to a similar or larger sod-rho transformation, even with a shorter hydrocarbon chain, than that of nonylamine. It must be noted that sample C9@SIM-1 also showed a significant structure change to rho despite its short functionalization extent (6.8\%). This may be explained by its solution in a more voluminous solvent (ethanol) as compared with hexylamine (water). 


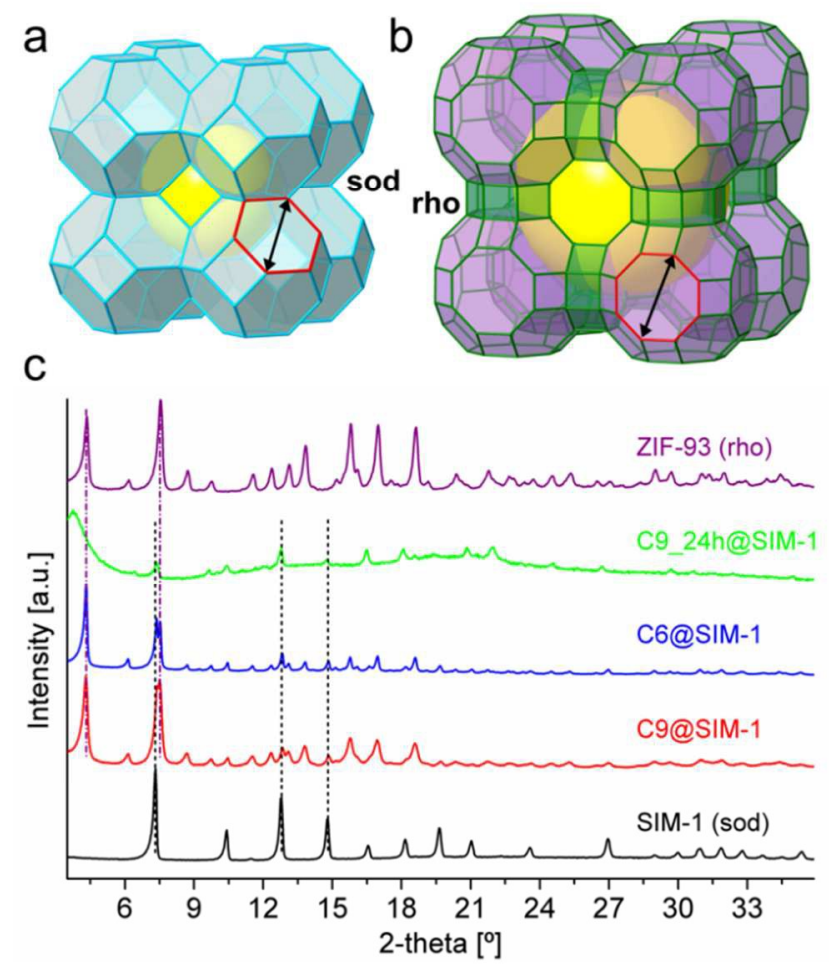

Figure 4. Rendering of the sod structure (a), composed of sod cages (blue), and the rho structure (b), made of lta (purple) and d8r (green) composite building units, drawn with Diamond software. ${ }^{47}$ Yellow spheres with the maximum diameter fitting in the cavity are shown. The 6-member (sod) and 8-member (rho) rings determining the limiting access entrances to the structures are also indicated. (c) XRD spectra of the pure ZIF-93 (rho) and SIM-1 (sod) powdered samples together with those of the amine-functionalized samples. A crystalline phase transformation from the sod structure is clearly observed as a consequence of the reaction with voluminous aliphatic-chain amines.

This structural alteration may have important consequences in the posterior HF membrane performance, because of a resultant aperture in the pore limiting diameter. Figure 4 shows the limiting ring apertures of the sod (6-membered) and rho (8-membered) structures. ${ }^{20,48}$ However, this crystalline phase alteration has not been reported in previous studies of MOF post-functionalization with amines, ${ }^{26,28}$ including SIM- $1 .{ }^{30}$ Neither was this phase transition reported in the ZIF-90 transformation into ZIF-92 by Caro et al. ${ }^{28}$ ZIF-90 (sod) has no competitive phase: the methyl group in position 2 in the 2-methylimidazolate ring supposedly prevents the formation of the rho-phase. ${ }^{35,37}$ However, SIM-1 (sod), with methyl and aldehyde groups in positions 4 and 5 , has a competitive construction, rho, so that changes in the reaction media (e.g. solvents and guest molecules) can induce this phase transition.

A completion of the imine-condensation reaction with SIM-1 powder was accomplished by increasing the reaction time up to $24 \mathrm{~h}$ at room temperature (sample C9_24h@SIM-1). The FTIR spectrum showed a $100 \%$ functionalization of SIM-1 aldehyde groups (Figure 3). However, $\mathrm{XRD}$ and later the $\mathrm{CO}_{2}$ adsorption isotherm (Figures 4 and $5 b$ ) revealed the above-mentioned partial destruction of the crystalline structure that led to an 
amorphous powder with a smaller SSA and a poor $\mathrm{CO}_{2}$ adsorption capacity. Its XRD pattern showed only crystalline remains of the sod structure. It can be deduced that the deep penetration of voluminous nonylamine molecules in the SIM-1 crystalline structure led first to a structural alteration (sod to rho) and then to the amorphization of the bulk material. In the same manner, only a $16.1 \mathrm{wt} \%$ residue was left from sample C9_24h@SIM-1 at $750{ }^{\circ} \mathrm{C}$ (Table 2 and Figure S1), lower than expected from a $100 \%$ functionalized SIM-1 (19.9 wt\%). This could be also related with the loss of crystallinity of the structure during the functionalization process. In consequence, in order to obtain a proper modification of the properties of the MOF HF membranes, functionalization with amines must be controlled so as to be only partially completed, thereby limited in the outermost layers of the supported MOF (the part in contact with the gas mixture to be separated) but not in the bulk of SIM-1.

Aguado et al. have also reported SIM-1 hydrophobization by means of the modification of alumina-supported SIM-1 powder with dodecylamine in methanol. ${ }^{30}$ The progress of the reaction was studied by NMR, yielding a 22-50\% conversion of the aldehyde groups depending on the support on which the MOF was prepared. However, no crystalline phase transition into ZIF-93 (rho) was observed although there was some loss of crystallinity and textural properties. It can be hypothesized that the DMF inside the non-activated SIM-1 pores, insoluble with $\mathrm{MeOH}$, prevented a bulk reaction and structural modification or damage, and that iminecondensation was limited to the crystal surface.

\section{$\underline{3.3 \text { Functionalized SIM-1@P84 }{ }^{\circledR} \text { HF membranes with tuned gas separation performance }}$}

\section{$\underline{\text { 3.3.1 Changes in textural properties }}$}

The modifications induced with the imine-condensation functionalization of the SIM-1 layer inside the P84 ${ }^{\circledR}$ supports, stepwise implemented in the microfluidic fabrication of the membranes, led to significant consequences for their performance. As observed above, a partial conversion of the crystalline phase together with a pore constriction resulting from the functionalization reaction must be taken into account when explaining the membrane separation behavior.

$\mathrm{N}_{2}$ adsorption isotherms at $77 \mathrm{~K}$ showed significant changes in the corresponding powders. Figure 5a and Table 3 show an increase in the pore volume and the specific surface area in the nonylamine-functionalized C9@SIM-1 derived from the sod conversion into the rho structure even at a low extent of reaction (6.8 \%). The pore volume gain from the structure transformation was not countervailed by the nonylamine chains introduced by means of the functionalization reaction. Thus the SSA of C9@SIM-1 increased from 591 to $687 \mathrm{~m}^{2} \cdot \mathrm{g}^{-1}$, still not as high as that of the ZIF-93 (pure rho phase), namely $765 \mathrm{~m}^{2} \cdot \mathrm{g}^{-1}$. In contrast, the reaction with hexylamine, where a $43 \%$ total conversion was calculated, led to a reduction of the accessible pore volume and SSA in sample C6@SIM-1 $\left(365 \mathrm{~m}^{2} \cdot \mathrm{g}^{-1}\right.$, see Figure 5a and Table 3). This is consistent with the penetration of the hexylamine chains into the SIM-1 structure countervailing the crystalline transition from sod to rho. 

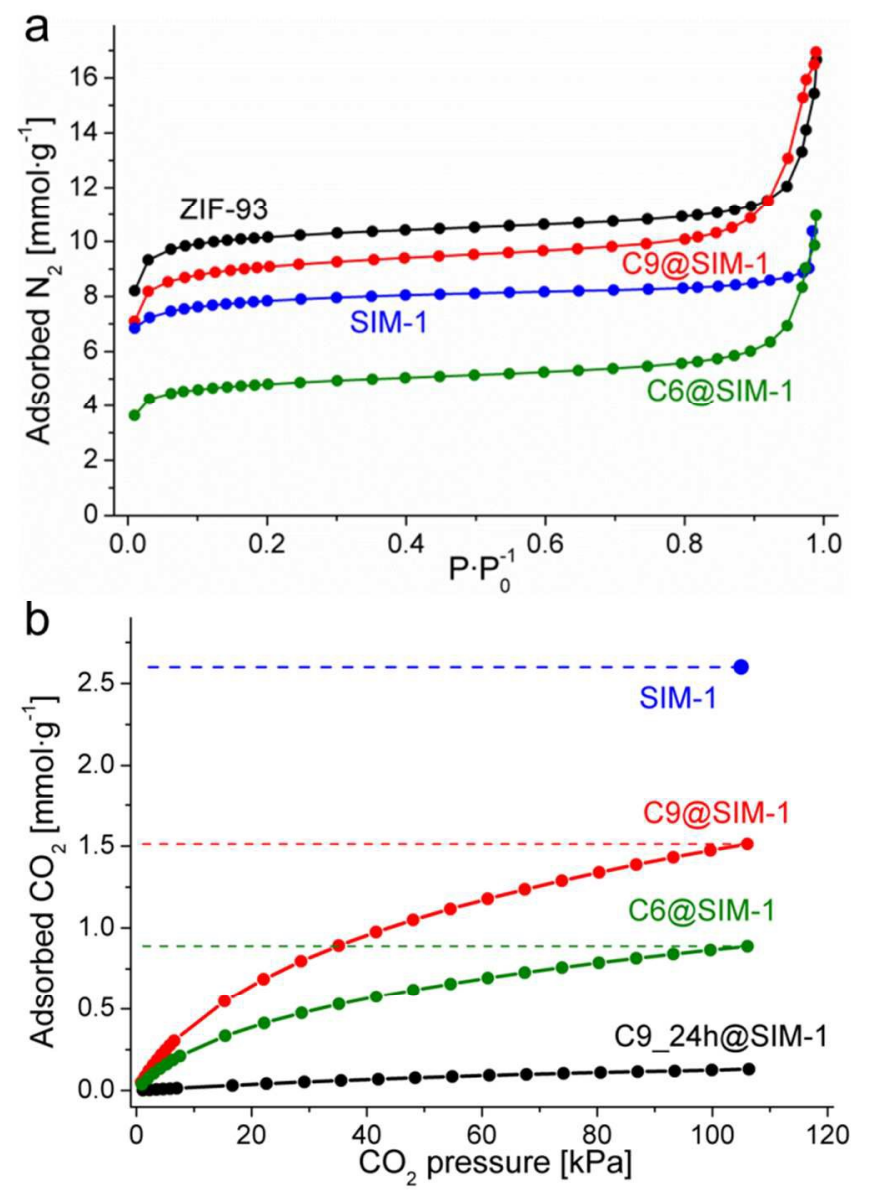

Figure 5. (a) $\mathrm{N}_{2}$ adsorption isotherms at $77 \mathrm{~K}$ of ZIF-93 (rho) and SIM-1 (sod) powders together with those of the amine-functionalized SIM-1 samples. (b) $\mathrm{CO}_{2}$ adsorption isotherms at $298 \mathrm{~K}$ of the functionalized SIM-1 samples with hexylamine and nonylamine. An averaged adsorption capacity of SIM-1 from data in the literature ${ }^{18,20,30}$ is also shown for comparison.

$\mathrm{CO}_{2}$ isotherms at $298 \mathrm{~K}$ showed a reduction in the adsorption capacity of the functionalized samples C9@ and C6@SIM-1 compared to that of as-prepared SIM-1 (Figure 5). This reduction depended on the extent of the functionalization reaction, so that the $43 \%$ reacted C6@SIM-1 only adsorbed 0.89 mmol $\mathrm{CO}_{2}$ per gram, whereas the $6.8 \%$ C9@SIM-1 adsorbed $1.5 \mathrm{mmol} \cdot \mathrm{g}^{-1}$ (Figure 5b and Table 3), less than half the $\mathrm{CO}_{2}$ adsorption capacity of SIM-1 at $303 \mathrm{~K}$ reported by Aguado et al. $\left(2.5-2.9 \mathrm{mmol} \cdot \mathrm{g}^{-1}\right) .{ }^{18,30}$ This is the case even after the $\mathrm{N}_{2}$ pore volume gain in the C9@SIM-1 mentioned above. Our hypothesis is that this phenomenon is related with a diminished $\mathrm{CO}_{2}$ affinity of the material, induced by the partial substitution during the functionalization reaction: a highly polar group (aldehyde) with a delocalized electric charge was substituted by a fully-saturated apolar hydrocarbon chain. ${ }^{49,50}$ The reduction in the polar moieties that strongly adsorb $\mathrm{CO}_{2}$ would lead to a faster $\mathrm{CO}_{2}$ diffusion through the structure of the functionalized SIM-1, independently from the modified crystalline phase (sod partially converted to rho in the functionalized samples). In fact, the $\mathrm{CO}_{2}$ adsorption capacity of these functionalized samples was still lower than that of ZIF-93 $\left(1.6 \mathrm{mmol} \cdot \mathrm{g}^{-1}\right) \mathrm{at}$ 1 bar reported by Morris et al. ${ }^{20}$ In contrast, at high pressures (up to 60 bar), ${ }^{20}$ and as reported by Biswal et 
al., ${ }^{37}$ higher $\mathrm{CO}_{2}$ adsorption capacities have been reported for the rho-structured ZIF than for the same ZIF with the sod structure because of the larger pore volumes of the former.

Table 3. Adsorption properties of the pure and functionalized ZIF samples.

\begin{tabular}{|c|c|c|c|}
\hline & $\begin{array}{c}\mathrm{SSA}^{a} \\
{\left[\mathrm{~m}^{2} \cdot \mathrm{g}^{-1}\right]}\end{array}$ & $\begin{array}{l}\text { Pore volume } \\
\qquad\left[\mathrm{cm}^{3} \cdot \mathrm{g}^{-1}\right]\end{array}$ & $\begin{array}{c}\mathrm{CO}_{2} \text { adsorbed }^{c} \\
{\left[\mathrm{mmol}^{c} \mathrm{~g}^{-1}\right]}\end{array}$ \\
\hline SIM-1 & 591 & 0.31 & $2.4-2.9^{18,20,30}$ \\
\hline C6@SIM-1 & 365 & 0.29 & 0.89 \\
\hline C9@SIM-1 & 687 & 0.53 & 1.5 \\
\hline C9_24h@SIM-1 & - & - & 0.12 \\
\hline ZIF-93 & 765 & 0.46 & $1.6^{20}$ \\
\hline
\end{tabular}

${ }^{a}$ Specific surface area obtained from the adjustment of the $\mathrm{N}_{2}$ isotherm at $77 \mathrm{~K}$ to the BET equation.

${ }^{b}$ Obtained from the $\mathrm{N}_{2}$ adsorption capacity at a 0.97 relative pressure.

${ }^{c} \mathrm{CO}_{2}$ adsorption capacity at $298 \mathrm{~K}$ and 1 bar.

Note also that sample C9_24h@SIM-1 showed a low $\mathrm{CO}_{2}$ adsorption $\left(0.12 \mathrm{mmol} \cdot \mathrm{g}^{-1}\right)$, related with the amorphization of the crystalline structure owing to the completion of the functionalization reaction, as discussed above. Damage to the crystalline structure during the functionalization reaction may also be related with this reduction in the SIM-1 adsorption capacity, as observed before. ${ }^{30}$

\section{$\underline{\text { 3.3.2 Gas separation performance of amine-functionalized SIM-1@P84 }{ }^{\circledR} \text { HF membranes }}$}

As described in the Experimental section, the P84 ${ }^{\circledR}$-supported SIM-1 membranes were identically functionalized by means of a sequential step in the microfluidic membrane fabrication. The corresponding amine solutions were micro-pumped in the lumen of the HF membranes. Thus, the changes observed in the amine-functionalized SIM-1 powders could also predict an influence in the gas separation performance of the resulting membranes. To verify the effects of the post-synthetic functionalization inside the HF membrane, XRD analysis was carried out (Figure S2). The sod to rho partial transformation can be observed, in a lower grade than that observed in the powder samples. Thereby, some mass transport constraint in the SIM-1 thin film compared to the powder can be deduced.

The SIM-1@P84 ${ }^{\circledR}$ membrane functionalization with nonylamine led to an increase in the permeances in the separation of all the studied gas mixtures, while the corresponding selectivities decreased. The results with the membrane C9@SIM-1@P84 ${ }^{\circledR}$ are shown in Table 4. As could be inferred from the previous analyses with the powdered SIM-1, larger gas permeances were obtained owing to the increase in the C9@SIM-1 microporous volume resulting from the alteration in the crystalline structure (see Figure 4). Likewise, the $\mathrm{CO}_{2}$ permeance was enhanced by a factor of 4.5 (3.5 to $15.7 \mathrm{GPU})$ after the C9-functionalization, because of a reduced $\mathrm{CO}_{2}$ adsorption affinity that favored a faster $\mathrm{CO}_{2}$ diffusion (Figure 5b). However, the crystalline phase transition from sod to rho implied a widening of the limiting pore diameter that reduced the molecular sieving effect of the MOF microporous structure in the gas separations. A significant fall in the separation selectivities was 
therefore observed when compared to the pristine membrane SIM-1@P84 ${ }^{\circledR}$ : the selectivities in the $\mathrm{H}_{2} / \mathrm{CH}_{4}$ and $\mathrm{He} / \mathrm{CH}_{4}$ mixtures, mostly controlled by the molecular exclusion of the large $\mathrm{CH}_{4}$ molecules (with kinetic diameter of $0.38 \mathrm{~nm}$ as compared to $0.29 \mathrm{~nm}$ and $0.33 \mathrm{~nm}$ for $\mathrm{H}_{2}$ and $\mathrm{CO}_{2}$, respectively), were reduced by approximately a half from 136 and 160 to 86 and 79 , respectively. Nevertheless, the $\mathrm{CO}_{2} / \mathrm{CH}_{4}$ mixture selectivity with the functionalized HF membrane C9@SIM-1@P84 ${ }^{\circledR}$ only decreased by $26 \%$ (from 38 to 28, see Tables 1 and 4). The selectivity drop in this mixture could have been partially hindered by an enhancement in the $\mathrm{CO}_{2}$ diffusion resulting from the SIM-1 functionalization with the apolar long-chain amines.

Table 4. Permeance and selectivity results obtained in the gas mixture permeation tests at $35^{\circ} \mathrm{C}$ using $\mathrm{C} 9$ @SIM$1 @ \mathbf{P 8 4}^{\circledR}$ fabricated membranes.

\begin{tabular}{c|c|c|c|c|c|c|} 
& \multicolumn{2}{c}{$\begin{array}{c}\text { Kinetic } \\
\text { Gas }\end{array}$} & \multicolumn{3}{c}{ Permeance } & \multicolumn{4}{c}{ Selectivity } \\
\cline { 3 - 7 } & diameter $[\AA]$ & {$\left[\mathbf{m o l} \cdot \mathbf{m}^{-2} \cdot \mathbf{s}^{-1} \cdot \mathbf{P a}^{-1}\right]$} & {$[\mathbf{G P U}]$} & $\mathbf{H}_{\mathbf{2}}$ & $\mathbf{C O}_{\mathbf{2}}$ & $\mathbf{C H}_{\mathbf{4}}$ \\
\hline $\mathbf{H e}$ & 2.6 & $1.5 \cdot 10^{-8}$ & 44.7 & $1.0^{a}$ & $2.9^{a}$ & 79.1 \\
\hline $\mathbf{H}_{\mathbf{2}}$ & 2.9 & $1.6 \cdot 10^{-8}$ & 46.6 & & 2.8 & 85.6 \\
\hline $\mathbf{C O}_{\mathbf{2}}$ & 3.3 & $5.3 \cdot 10^{-9}$ & 15.7 & & & 27.8 \\
\hline $\mathbf{C H}_{\mathbf{4}}$ & 3.8 & $1.9 \cdot 10^{-10}$ & 0.6 & & & \\
\hline
\end{tabular}

${ }^{a}$ Ideal selectivities calculated as the ratio of gas permeance data in the studied mixtures.

The C9-functionalization of the SIM-1 layer in a newly fabricated HF membrane enhanced the gas permeances because of an enlargement of the limiting-pore size resulting from the crystalline phase conversion from sod to rho. Therefore, the C9@SIM-1@P84 ${ }^{\circledR}$ membrane became more permeable in the gas mixture separations (e.g. the $\mathrm{He}$ and $\mathrm{H}_{2}$ permeances increased from 15 and 13 to 45 and $47 \mathrm{GPU}$, respectively), while the molecular sieving effect was somewhat diminished. Only the $\mathrm{CO}_{2} / \mathrm{CH}_{4}$ separation selectivity was less affected than that for mixtures with $\mathrm{He}$ and $\mathrm{H}_{2}$, which can be explained by a reduction in the $\mathrm{CO}_{2}$ adsorption affinity in the $\mathrm{C} 9 @$ SIM-1 material, as shown above.

The performance of membrane C9@SIM-1@P84 ${ }^{\circledR} \mathrm{HF}$ in the $\mathrm{CO}_{2} / \mathrm{CH}_{4}$ mixture separation was close to that predicted by Ray et al. ${ }^{48}$ for the rho-ZIF-93 material (selectivity of 26.7) and improved the results obtained in our previous work: a $\mathrm{CO}_{2} / \mathrm{CH}_{4}$ separation selectivity of 17 using a P84 ${ }^{\circledR}$-supported ZIF-93 HF membrane. ${ }^{22}$ This comparison is shown in Figure 6 together with other data arising from MOF@HF membranes. Therefore, a combination of a mixed sod-rho structure with a partially $\mathrm{C} 9$-imine functionalized surface proved to be positive in the $\mathrm{CO}_{2} / \mathrm{CH}_{4}$ separation. This showed a good permeance-selectivity balance compared to the only sod membrane, SIM-1@P84 ${ }^{\circledR}$ (with a $\mathrm{CO}_{2} / \mathrm{CH}_{4}$ separation selectivity of 37.7 , Table 1), because of its enhanced $\mathrm{CO}_{2}$ transport. The permeance-selectivity balance is the main threshold to be overcome in membrane technology. ${ }^{51}$ Indeed, this better performance in terms of permeability-selectivity of the functionalized MOF HF membranes highlights the advantages of the new methodology based on the microfluidics implemented here: i) the functionalization can be sequentially in situ carried out following the MOF crystallization; ii) it minimizes the possibility of contamination of the membrane sample with the 
surrounding atmosphere; iii) it saves reactants, as demonstrated in previous reports; ${ }^{42}$ and, finally, iv) it allows an easier scaling up membrane preparation by parallelization, ${ }^{17,52}$ also taking advantage of the intrinsic high efficiency and compactness of the membrane conformation as HF. Moreover, the fabricated membranes showed the robustness necessary to compensate the possible stress derived from the increase in the MOF unit cell volume during the sod to rho structural transformation. This can be related with the advantageous compatibility between the MOF layer and the flexible polymeric HF support because of their common organic characters.

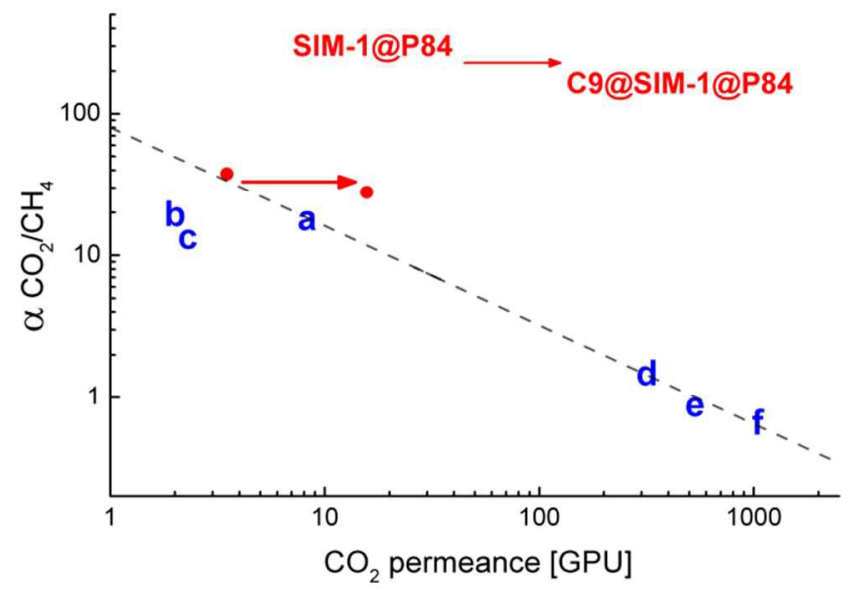

Figure 6. Gas permeance and selectivity comparison of the $\mathrm{CO}_{2} / \mathrm{CH}_{4}$ mixture separation performance displayed by HF membranes SIM-1@P84 ${ }^{\circledR}$ and C9@SIM-1@P84 ${ }^{\circledR}$. Other MOF-supported polymeric HF membrane data are shown for comparison: ZIF-8@ and ZIF-93@P84 ${ }^{\circledR}$ (a, b), ${ }^{45}$ ZIF-7@PSF $(c)^{42}$, ZIF-90@TORLON ${ }^{\circledR}\left(\right.$ d $^{31}$ and NH $^{2-}$ MIL-53@ (e) $)^{53}$ and HKUST-1@PVDF (f). ${ }^{54}$ An upper bound adjustment to the presently-available experimental data is shown as a guide to the eye.

The functionalization carried out with water-soluble hexylamine led to the HF membrane C6@SIM-1@P84 ${ }^{\circledR}$. In this case, hexylamine showed a high reactivity with the co-polyimide P84 ${ }^{\circledR}$ support. This reaction has been widely reported in the literature: the degree of cross-linking grade of $\mathrm{P} 84^{\circledR}$ can be increased with a di-amine with the aim of making the polymer more resistant to organic solvents. ${ }^{55-59}$ The amine group is able to react with the imide-ring in $\mathrm{P} 84^{\circledR}$, thus breaking the nitrogen-carbonyl bond, as shown in Figure S3a. This undesired reaction took place in the SIM-1@P84 ${ }^{\circledR} \mathrm{HF}$ membrane during its functionalization with hexylamine, simultaneously with the imine-condensation in the supported-SIM-1 layer, leading to a wreckage of the $\mathrm{P} 84^{\circledR}$ structure. The resulting membrane became extremely stiff, showing a poor gas separation performance $\left(\mathrm{a} \mathrm{H}_{2} / \mathrm{CH}_{4}\right.$ separation selectivity of 6.0$)$ and minimum durability due to its brittleness. As observed in the bulk powder functionalization reaction, a higher amine-P84 ${ }^{\circledR}$ reaction extent was obtained with water-dissolved C6 than with ethanol-dissolved C9, maybe due to the smaller molecular size of the solvent. As a probe, a P84 ${ }^{\circledR} \mathrm{HF}$ membrane was allowed to react for $24 \mathrm{~h}$ with the C6- and C9-amine solutions used above. The FTIR spectra of the resulting samples are shown in Figure S3b. Although the ethanoldissolved nonylamine reaction with the $\mathrm{P} 84^{\circledR}$ polymer cannot be ignored, the reactivity was much lower than 
within the water-dissolved hexylamine media, where the $\mathrm{P} 84^{\circledR}$ polymer structure was seriously damaged after $24 \mathrm{~h}$. The imide bands $\left(1360,1713\right.$ and $\left.1778 \mathrm{~cm}^{-1}\right)$ disappeared in the C6_24h@P84 ${ }^{\circledR}$ spectrum, while the peaks relating to the amide functional group emerged $\left(1547\right.$ and $\left.1644 \mathrm{~cm}^{-1}\right){ }^{56}$

\section{Conclusions}

ZIF SIM-1, with a pendant aldehyde group, can be functionalized with an amine under mild conditions. This functionalization reaction has here been followed through FTIR, XRD, TGA and $\mathrm{N}_{2}$ and $\mathrm{CO}_{2}$ adsorption of the powdered material. Although limited extents of the reaction were obtained with both hexyl- and nonylamine (conversion of the aldehyde to imine groups with pendant aliphatic chains in the SIM-1 structure), a crystalline phase alteration was observed. The SIM-1 sod structure was partially transformed into a wider rho likely to accommodate the voluminous amine groups introduced (perhaps with a template role). Only short functionalization times were applied, since high extents of reaction led to a complete loss of crystallinity of the ZIF. Moreover, amine-functionalized SIM-1 showed significant changes in its textural properties, and a reduced affinity for $\mathrm{CO}_{2}$ adsorption. These changes in the SIM-1 structure affected the gas separation performance of the resulting membranes fabricated with this MOF.

The nonylamine-functionalized SIM-1@P84 ${ }^{\circledR}$ membrane showed higher gas permeances but lower selectivities compared to the as-made SIM-1@P84 ${ }^{\circledR}$ HF membranes. These are the first membranes fabricated with this MOF to be reported with high separation selectivities, in this case prepared on the inner side of HF supports by microfluidics. The separation selectivities for $\mathrm{He} / \mathrm{CH}_{4}$ and $\mathrm{H}_{2} / \mathrm{CH}_{4}$ reduced by about half from 160 and 136, respectively, in the case of the SIM-1@P84 ${ }^{\circledR}$ membranes to 79 and 86 in the case of the C9@SIM-1@P84 ${ }^{\circledR}$ membrane. This was the result of a decreased molecular sieving effect caused by the crystalline phase transformation described above. However, the $\mathrm{CO}_{2} / \mathrm{CH}_{4}$ separation selectivity decreased by only $26 \%$ (from 38 to 28 ), the $\mathrm{CO}_{2}$ permeance increasing from 4 to 16 GPU. This was attributed to the withdrawal of the free electrical charge of the aldehyde polar group through the imine-condensation reaction, which led to enhanced $\mathrm{CO}_{2}$ diffusion through the $\mathrm{C} 9 @$ SIM-1 structure. Hexylamine showed a high undesired reactivity with the $\mathrm{P} 84^{\circledR}$ polymer in the HF support and led to the collapse of the membrane.

Finally, although several post-synthetic functionalization reactions with ZIFs have been previously reported in the literature, a direct relationship of these with the changes in the gas separation performance has been found in this work. An in situ sequential post-synthetic reaction was easily accomplished in the HF membrane fabricated by microfluidics. The easily-controllable additional step comprising the functionalization led to a permeance-selectivity performance balancing the molecular sieving effect through the constricted pores of the MOF and adsorption phenomena in the resulting membrane. This technique stands out for its versatility and suitability for up-scaling by means of parallelization, and is also environmentally friendly in terms of efficiency (HF membrane modules offer the highest area to volume ratio) and reactant savings.

\section{$\underline{\text { 5. Acknowledgements }}$}


Financial support (MAT2013-40556-R) from the Spanish MINECO, the Aragón Government (DGA, T05) and the European Social Fund is gratefully acknowledged. We also acknowledge the use of the Servicio General de Apoyo a la Investigación-SAI (Universidad de Zaragoza). F.C.-B. acknowledges his DGA predoctoral fellowship. All the microscopy work was done in the Laboratorio de Microscopías Avanzadas at the Instituto de Nanociencia de Aragón (LMA-INA). The authors acknowledge the LMA-INA for offering access to their instruments and expertise.

\section{Notes and references}

1. R. Banerjee, A. Phan, B. Wang, C. Knobler, H. Furukawa, M. O'Keeffe and O. M. Yaghi, Science, 2008, 319, 939-943.

2. O. M. Yaghi, M. O'Keeffe, N. W. Ockwig, H. K. Chae, M. Eddaoudi and J. Kim, Nature, 2003, 423, 705-714.

3. J. Gascon, F. Kapteijn, B. Zornoza, V. Sebastian, C. Casado and J. Coronas, Chem. Mater., 2012, 24, 2829-2844.

4. Y. Peng, Y. Li, Y. Ban, H. Jin, W. Jiao, X. Liu and W. Yang, Science, 2014, 346, 1356-1359.

5. K. Sumida, D. L. Rogow, J. A. Mason, T. M. McDonald, E. D. Bloch, Z. R. Herm, T. H. Bae and J. R. Long, Chem. Rev., 2012, 112, 724-781.

6. C. Montoro, E. García, S. Calero, M. A. Pérez-Fernández, A. L. López, E. Barea and J. A. R. Navarro, J. Mater. Chem., 2012, 22, 10155-10158.

7. J. Yang, F. Zhang, H. Lu, X. Hong, H. Jiang, Y. Wu and Y. Li, Angew. Chem. Int. Ed., 2015, 54, 10889-10893.

8. E. López-Maya, C. Montoro, L. M. Rodríguez-Albelo, S. D. Aznar Cervantes, A. A. Lozano-Pérez, J. L. Cenís, E. Barea and J. A. R. Navarro, Angew. Chem., Int. Ed., 2015, 54, 6790-6794.

9. P. Horcajada, T. Chalati, C. Serre, B. Gillet, C. Sebrie, T. Baati, J. F. Eubank, D. Heurtaux, P. Clayette, C. Kreuz, J. S. Chang, Y. K. Hwang, V. Marsaud, P. N. Bories, L. Cynober, S. Gil, G. Ferey, P. Couvreur and R. Gref, Nat. Mater., 2010, 9, 172-178.

10. J. A. Thompson, C. R. Blad, N. A. Brunelli, M. E. Lydon, R. P. Lively, C. W. Jones and S. Nair, Chem. Mater., 2012, 24, 1930-1936.

11. J. A. Thompson, J. T. Vaughn, N. A. Brunelli, W. J. Koros, C. W. Jones and S. Nair, Microporous Mesoporous Mater., 2014, 192, 43-51. 
12. K. Eum, K. C. Jayachandrababu, F. Rashidi, K. Zhang, J. Leisen, S. Graham, R. P. Lively, R. R. Chance, D. S. Sholl, C. W. Jones and S. Nair, J. Am. Chem. Soc., 2015, 137, 4191-4197.

13. A. Marti, M. Van and K. J. Balkus, J. Porous Mater., 2014, 1-14.

14. H. Fei, J. F. Cahill, K. A. Prather and S. M. Cohen, Inorg. Chem., 2013, 52, 4011-4016.

15. F. G. Xi, H. Liu, N. N. Yang and E. Q. Gao, Inorg. Chem., 2016, 55, 4701-4703.

16. R. J. Marshall, T. Richards, C. L. Hobday, C. F. Murphie, C. Wilson, S. A. Moggach, T. D. Bennett and R. S. Forgan, Dalton Trans., 2016, 45, 4132-4135.

17. C. Zhang and W. J. Koros, J. Phys. Chem. Lett., 2015, 6, 3841-3849.

18. S. Aguado, C. H. Nicolas, V. Moizan-Basle, C. Nieto, H. Amrouche, N. Bats, N. Audebrand and D. Farrusseng, New J. Chem., 2011, 35, 41-44.

19. S. Aguado, J. Canivet and D. Farrusseng, Chem. Commun., 2010, 46, 7999-8001.

20. W. Morris, N. He, K. G. Ray, P. Klonowski, H. Furukawa, I. N. Daniels, Y. A. Houndonougbo, M. Asta, O. M. Yaghi and B. B. Laird, J. Phys. Chem. C, 2012, 116, 24084-24090.

21. W. Morris, B. Leung, H. Furukawa, O. K. Yaghi, N. He, H. Hayashi, Y. Houndonougbo, M. Asta, B. B. Laird and O. M. Yaghi, J. Am. Chem. Soc., 2010, 132, 11006-11008.

22. F. Cacho-Bailo, G. Caro, M. Etxeberría-Benavides, O. Karvan, C. Tellez and J. Coronas, Chem. Commun., 2015, 51, 11283-11285.

23. T. Yang and T. S. Chung, J. Mater. Chem. A, 2013, 1, 6081-6090.

24. D. Hua, Y. K. Ong, Y. Wang, T. Yang and T. S. Chung, J. Membr. Sci., 2014, 453, 155-167.

25. R. Banerjee, H. Furukawa, D. Britt, C. Knobler, M. O'Keeffe and O. M. Yaghi, J. Am. Chem. Soc., 2009, 131, 3875-3877.

26. W. Morris, C. J. Doonan, H. Furukawa, R. Banerjee and O. M. Yaghi, J. Am. Chem. Soc., 2008, 130, 12626-12627.

27. J. Jiang, Y. Zhao and O. M. Yaghi, J. Am. Chem. Soc., 2016, 138, 3255-3265.

28. A. S. Huang and J. Caro, Angew. Chem. Int. Ed., 2011, 50, 4979-4982.

29. L. Q. Yu, C. X. Yang and X. P. Yan, J. Chromatogr. A, 2014, 1343, 188-194. 
30. S. Aguado, J. Canivet and D. Farrusseng, J. Mater. Chem., 2011, 21, 7582-7588.

31. A. J. Brown, J. R. Johnson, M. E. Lydon, W. J. Koros, C. W. Jones and S. Nair, Angew. Chem. Int. Ed., 2012, 51, 10615-10618.

32. A. J. Brown, N. A. Brunelli, K. Eum, F. Rashidi, J. R. Johnson, W. J. Koros, C. W. Jones and S. Nair, Science, 2014, 345, 72-75.

33. F. K. Shieh, S. C. Wang, S. Y. Leo and K. C. W. Wu, Chem. Eur. J., 2013, 19, 11139-11142.

34. H. T. Kwon and H. K. Jeong, Chem. Commun., 2013, 49, 3854-3856.

35. X. C. Huang, Y. Y. Lin, J. P. Zhang and X. M. Chen, Angew. Chem., Int. Ed., 2006, 45, 1557-1559.

36. K. S. Park, Z. Ni, A. P. Cote, J. Y. Choi, R. D. Huang, F. J. Uribe-Romo, H. K. Chae, M. O'Keeffe and O. M. Yaghi, Proc. Natl. Acad. Sci. U. S. A., 2006, 103, 10186-10191.

37. B. P. Biswal, T. Panda and R. Banerjee, Chem. Commun., 2012, 48, 11868-11870.

38. J. Quirós, K. Boltes, S. Aguado, R. Guzman de Villoria, J. J. Vilatela and R. Rosal, Chem. Eng. J., 2015, 262, 189-197.

39. M. Baias, A. Lesage, S. Aguado, J. Canivet, V. Moizan-Basle, N. Audebrand, D. Farrusseng and L. Emsley, Angew. Chem., Int. Ed., 2015, 54, 5971-5976.

40. F. Cacho-Bailo, I. Matito-Martos, J. Perez-Carbajo, M. Etxeberria-Benavides, O. Karvan, V. Sebastian, S. Calero, C. Tellez and J. Coronas, Chem. Sci., 2017, 8, 325-333.

41. F. Maya, C. Palomino Cabello, S. Clavijo, J. M. Estela, V. Cerda and G. Turnes Palomino, Chem. Commun., 2015, 51, 8169-8172.

42. F. Cacho-Bailo, S. Catalán-Aguirre, M. Etxeberría-Benavides, O. Karvan, V. Sebastian, C. Téllez and J. Coronas, J. Membr. Sci., 2015, 476, 277-285.

43. C. Y. Feng, K. C. Khulbe, T. Matsuura and A. F. Ismail, Sep. Purif. Technol., 2013, 111, 43-71.

44. Y. Dai, J. R. Johnson, O. Karvan, D. S. Sholl and W. J. Koros, J. Membr. Sci., 2012, 401-402, 7682.

45. F. Cacho-Bailo, G. Caro, M. Etxeberria, O. Karvan, C. Tellez and J. Coronas, $R S C A d v ., 2016,6$, 5881-5889. 
46. Y. Yampolskii, Macromolecules, 2012, 45, 3298-3311.

47. Diamond - Crystal and Molecular Structure Visualization; Crystal Impact - Dr. H. Putz \& Dr. K. Brandenburg GbR, Kreuzherrenstr. 102, 53227 Bonn, Germany; http://www.crystalimpact.com/diamond.

48. K. G. Ray, D. L. Olmsted, J. M. R. Burton, Y. Houndonougbo, B. B. Laird and M. Asta, Chem. Mater., 2014, 26, 3976-3985.

49. O. Benson, I. da Silva, S. P. Argent, R. Cabot, M. Savage, H. G. W. Godfrey, Y. Yan, S. F. Parker, P. Manuel, M. J. Lennox, T. Mitra, T. L. Easun, W. Lewis, A. J. Blake, E. Besley, S. Yang and M. Schröder, J. Am. Chem. Soc., 2016, 138, 14828-14831.

50. E. Atci and S. Keskin, J. Phys. Chem. C, 2012, 116, 15525-15537.

51. L. M. Robeson, J. Membr. Sci., 2008, 320, 390-400.

52. B. P. Biswal, A. Bhaskar, R. Banerjee and U. K. Kharul, Nanoscale, 2015, 7, 7291-7298.

53. W. Li, P. Su, G. Zhang, C. Shen and Q. Meng, J. Membr. Sci., 2015, 495, 384-391.

54. Y. Mao, J. Li, W. Cao, Y. Ying, L. Sun and X. Peng, ACS Appl. Mater. Interfaces, 2014, 6, 44734479 .

55. K. Vanherck, P. Vandezande, S. O. Aldea and I. F. J. Vankelecom, J. Membr. Sci., 2008, 320, 468476.

56. K. Vanherck, G. Koeckelberghs and I. F. J. Vankelecom, Prog. Polym. Sci., 2013, 38, 874-896.

57. J. Gao, S. P. Sun, W. P. Zhu and T. S. Chung, J. Membr. Sci., 2014, 452, 300-310.

58. C. Ba, J. Langer and J. Economy, J. Membr. Sci., 2009, 327, 49-58.

59. S. Sorribas, P. Gorgojo, C. Tellez, J. Coronas and A. G. Livingston, J. Am. Chem. Soc., 2013, 135, 15201-15208. 


\section{Graphical Abstract}

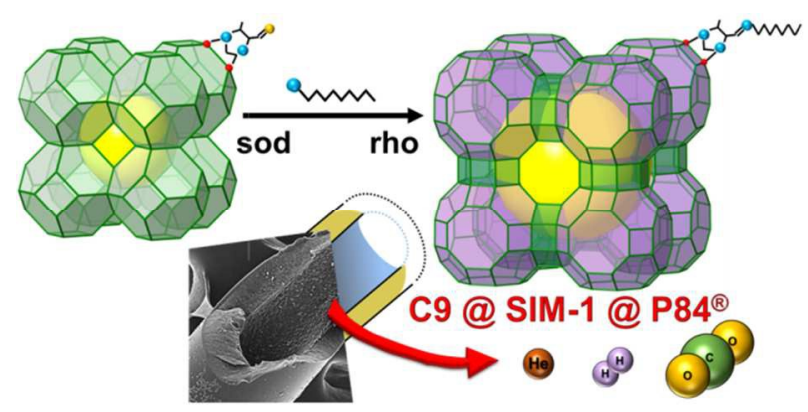

For Table of Contents Only

"SIM-1(sod) to ZIF-93(rho) structural change through post-synthetic amine-functionalization tunes the membrane performance in the separation of gas mixtures." 